\title{
CORRECTION
}

\section{Correction to: Measured Phenology Response of Unchanged Crop Varieties to Long-Term Historical Climate Change}

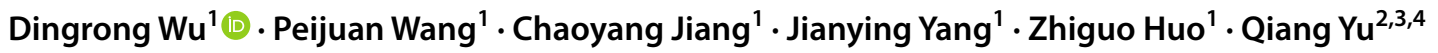

Published online: 28 January 2019

(c) Springer Nature Switzerland AG 2019

\section{Correction to: International Journal of Plant Production https://doi.org/10.1007/s42106-018-0033-z}

The original version of this article unfortunately contained mistakes. Table 1 was incorrect. The corrected Table 1 is given below.

Table 1 Information of sites which the planted variety kept unchanged for at least 15 years

\begin{tabular}{lrlll}
\hline Site & Elevation $(\mathrm{m})$ & Year of planting & $\begin{array}{l}\text { Number of } \\
\text { planting years }\end{array}$ & Variety name \\
\hline Winter wheat & & & & \\
Changzhi & 991.8 & $1988-2004$ & 17 & CZ648 \\
Hancheng & 458.1 & $1991-1994,1996-2003,2006-2009$ & 15 & XY6 \\
Jincheng & 752.6 & $1981-1986,1989-1992,1994-1998$, & 21 & 5819 \\
& & $2001,2003-2006$ & & \\
Huanghua & 6.6 & $1989-2011$ & 23 & 71321 \\
Laizhou & 48.4 & $1990-2010$ & 21 & YN15 \\
Fushan & 53.9 & $1987-2011$ & 26 & YN15 \\
Tianshui & 1141.6 & $1991-2009$ & 19 & 7464 \\
Single rice & & & & QG \\
Tonghua & 380.1 & $1985-2010$ & 26 & SY397 \\
Muling & 266.3 & $1994-1995,1997-2011$ & 16 & \\
Spring maize & & & & DN248 \\
Jiamusi & 82.0 & $1992-2002,2004-2010$ & 18 & TD4 \\
Meihekou & 340.5 & $1986-1993,1995-2001$ & 15 & JD101 \\
Tonghua & 380.1 & $1983-2002$ & 20 & \\
\hline
\end{tabular}

The original article can be found online at https://doi.org/10.1007/ s42106-018-0033-z.

Qiang Yu

yuq@igsnrr.ac.cn

1 State Key Laboratory of Severe Weather (LASW), Chinese Academy of Meteorological Sciences, Beijing 100081, China

2 State Key Laboratory of Soil Erosion and Dryland Farming on the Loess Plateau, Northwest A\&F University, Yangling 712100, China

3 Plant Functional Biology and Climate Change Cluster, University of Technology Sydney, Broadway, PO Box 123, Ultimo, NSW 2007, Australia

4 College of Resources and Environment, University of Chinese Academy of Science, Beijing 100049, China 